\title{
Vagal input to the enteric nervous system
}

\author{
T L Powley
}

\section{Department of Psychological Science, Purdue University, West Lafayette, Indiana 47907 , USA T L Powley \\ Correspondence to: Dr T L Powley. powleytl@psych.purdue.edu}

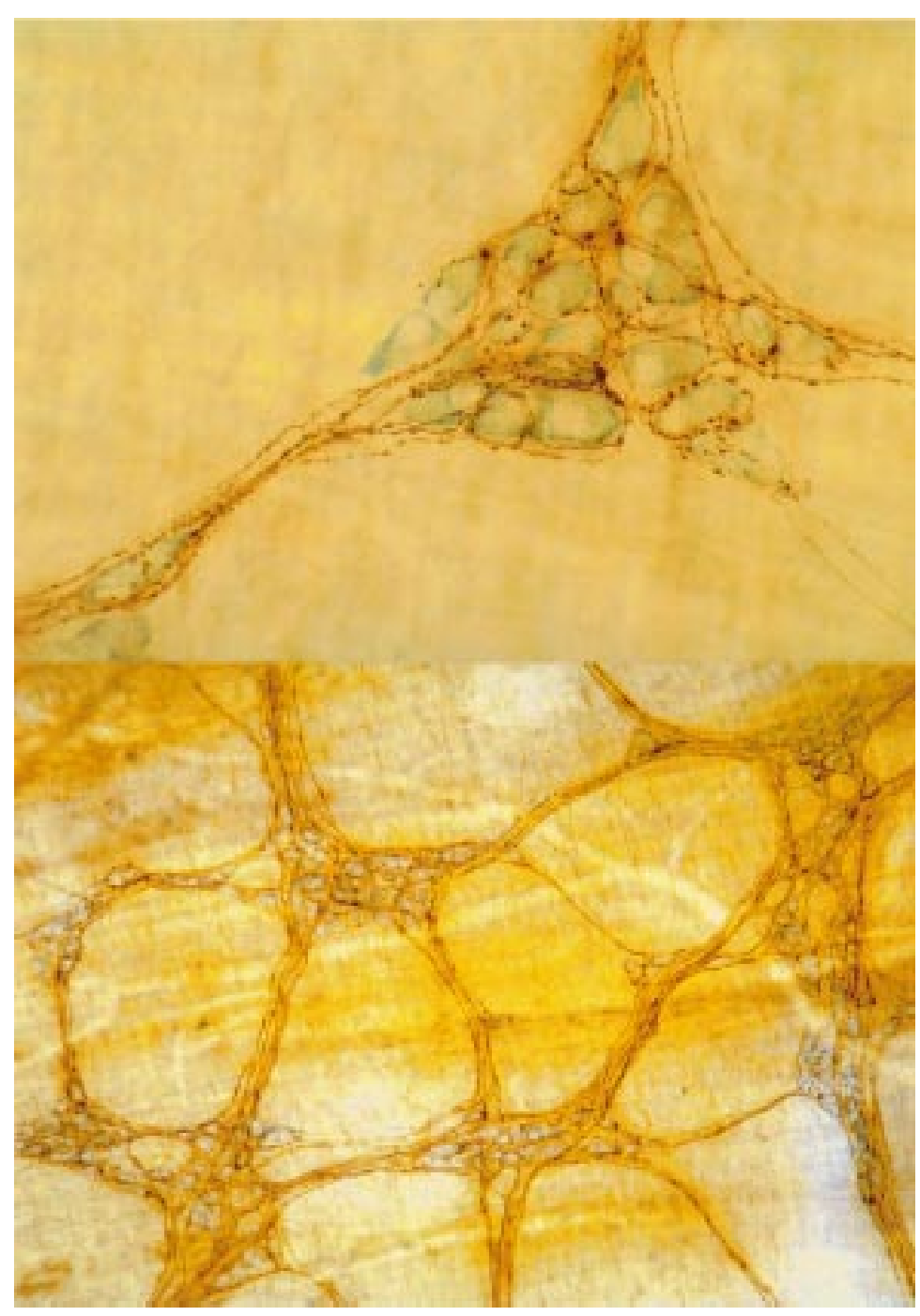

Figure 1 Labelled vagal motor axons (brown, Phaseolus vulgaris-leucoagglutinin) contacting myenteric ganglionic neurones (blue, Cuprolinic blue counterstaining) in a wholemount of the rat stomach. Top: High power view illustrating vagal preganglionic projections to a single myenteric ganglion. Vagal axons make presumptive contacts (that is, establish varicosities near) with almost all gastric enteric neurones. Bottom: Low power view illustrating that vagal preganglionic axons form an extensive network enmeshing each gastric myenteric ganglion with varicose terminals (see Holst et al for additional examples ${ }^{4}$.

\section{Background}

The enteric nervous system (ENS) of the tract has traditionally been largely independently of the brain and spinal cord. Because of this postulated autonomy, the ENS has even been characterised as the "little brain" or the "second brain." Recent neural tracing studies, however, challenge this canon of ENS autonomy.

The view that the ENS is independent can be traced to several types of observation. Early research on the relationship of the ENS to the central nervous system (CNS) noted that the
ENS could support motor activity and a number of local reflexes when the viscera were decentralised or isolated. Langley (1922), ${ }^{1}$ in his seminal analyses, reinforced the idea of a largely autonomous ENS by noting that the preganglionic axons projecting to the ENS were far outnumbered by the multitude of enteric neurones in the viscera. In the case of the vagus nerve in particular, Langley envisioned a few specialised "mother cells" or "vagal cells" in the ENS that were contacted by preganglionic inputs and that then distributed the relatively small number of inputs to a bigger group of effector neurones within the enteric plexuses. In a related but more modern view, Wood $(1987)^{2}$ suggested that autonomic preganglionics might project to "command neurones" within the ENS. ${ }^{3}$ On the sensory side, early observations also noted that individuals with intact peripheral nerves connecting the ENS and CNS had little awareness of visceral afferent feedback.

Although the view of an all-but-autonomous ENS still tends to dominate thinking and teaching about the innervation of the gastrointestinal tract, it should be stressed that the concept is based on inconclusive premises. For example, although the spinal cord separated from the brain still supports a number of reflexes, it is not typically inferred that the cord normally operates independently of the brain. Although preganglionic axons may be outnumbered by enteric neurones, it is problematic to gauge preganglionic influences or organisation without information about ramifications or divergence of the efferents. Although much visceral afferent traffic does not reach awareness, it does not follow that afferent feedback is limited. Furthermore, it might be noted that the traditional extrapolations arguing for ENS autonomy tend to ignore the lessons from psychosomatic medicine.

Historically, then, the ideas of ENS autonomy have been based largely on indirect and/or relatively ambiguous arguments. These conclusions have been especially durable because of an absence of more direct evidence contradicting the assumptions. It has been particularly difficult to evaluate the autonomy idea structurally because of limitations of neuroanatomical techniques. Advances in neural tracing technologies in the past few years, however, have now made it practical to collect structural observations that directly address the extent of the interconnections between the ENS and CNS.

The present talk summarises a series of recent experiments using such new tracing strategies. The results question the model of

Abbreviations used in this paper: ENS, enteric nervous system; CNS, central nervous system. 


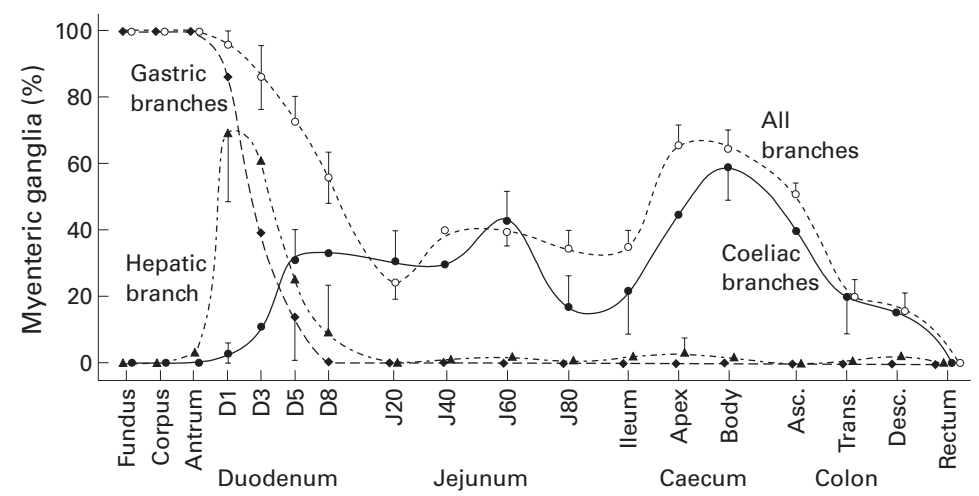

Figure 2 A summary of the percentage of all myenteric ganglia at different levels of the gastrointestinal tract that were innervated by DiI labelled axons after the tracer was injected into the dorsal motor nucleus of the vagus. "All branches" refers to data from a group of animals in which all vagal branches innervating the abdomen were left intact, thus the "all branches" function provides the best estimate of the extent of vagal motor projections to the myenteric ganglia of the gut. "Gastric," "hepatic," and "coeliac" branches refer to additional groups of animals in which only the designated branch of the abdominal vagus was left intact to transport tracer into the gastrointestinal tract (see Berthoud et al ${ }^{10}$ ).

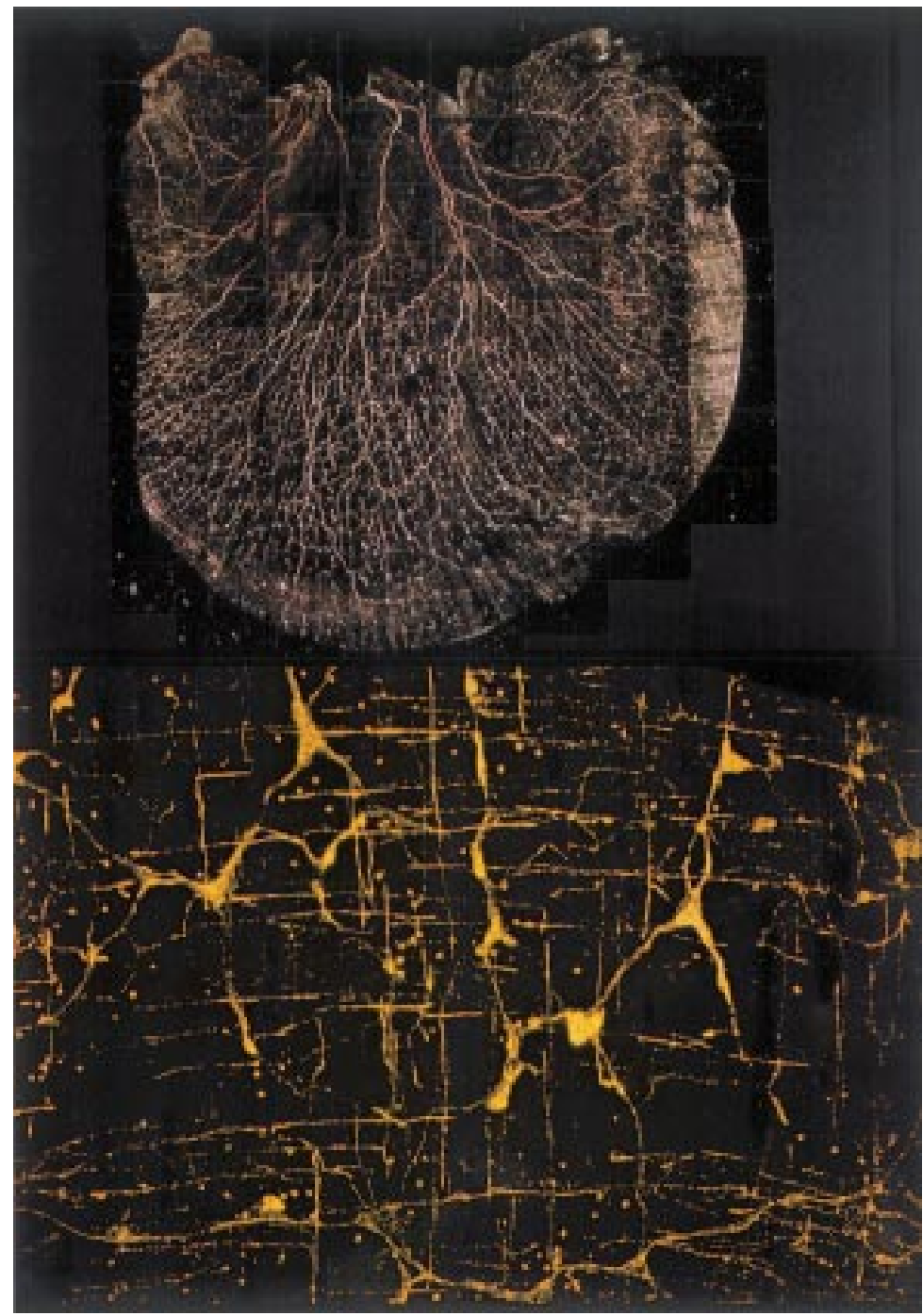

Figure 3 Labelled vagal afferent or sensory axons (gold, wheat germ agglutinin-horseradish peroxidase) in a wholemount of the rat stomach. Top: Low power montage of the muscle wall of the rat stomach illustrating the extensive network of afferent fibres and endings supplying the stomach. The pylorus is in the upper left corner of the wholemount; the forestomach is to the right; the greater curvature is at the bottom of the specimen. Bottom: High power view of vagal afferents innervating the muscle wall of the stomach. At this magnification, both vagal intraganglionic laminar endings (leaf shaped profiles consisting of plates of terminal puncta) and intramuscular arrays (long straight free nerve endings paralleling both the circular (vertical orientation) and longitudinal

(horizontal orientation) muscle fibres) are seen (see Phillips et al for additional examples ${ }^{7}$ ).
ENS autonomy and suggest that enteric neurones are enmeshed in a neural network that extends from the CNS into the gut.

\section{Methods}

To directly examine the connections between CNS outflow and the ENS, we injected anterograde neural tracers, including Phaseolus vulgaris-leucoagglutinin, ${ }^{4} \mathrm{DiI},{ }^{56}$ cholera toxin B subunit-horseradish peroxidase (unpublished), wheat germ agglutinin-horseradish peroxidase, ${ }^{7}$ and conjugated dextrans ${ }^{8}$ into brain stem pools of vagal motor neurones and prepared the stomach and duodenum as wholemounts. ${ }^{9}$ In some series, limited injections were used to label only a few neurones thus making it possible to trace the course in individual axons; in other series larger injections were used to label the entire population of neurones innervating visceral targets. The projections, including collateralisation and divergence, of individual axons innervating the ENS were then examined with both light and confocal microscopy and digitised.

\section{Results}

The picture emerging from these tracer studies is at variance with the concept of ENS autonomy. On reaching the enteric plexuses, individual vagal axons ramify extensively and widely, contacting large numbers of enteric neurones. When larger injections of tracer designed to label as many vagal preganglionic neurones as possible are used, the motor axons are seen to form a network of varicose endings investing almost all neurones of the myenteric plexus of the stomach and proximal small intestine (figs 1,2 ). Structurally, the pattern of vagal projections would appear to be more adequately described as a network rather than an impoverished input to a limited number of specialised mother cells or command neurones.

Independent recent electrophysiological experiments examining the responses of individual myenteric neurones of the stomach after vagal stimulation ${ }^{11}$ also substantiate the existence of extensive vagal efferent projections to the gastric myenteric plexus.

Another series of experimental observations using new neural tracing techniques also underscores the importance of reconsidering the traditional view of an independent ENS. In experiments paralleling those just summarised for vagal efferents, we have used injections of neural tracers into the nodose ganglia to label vagal afferent terminals in the wall of the gut. Vagal afferent axons (which are also vastly outnumbered by the quantity of enteric neurones in the gut) ramify extensively, filling the enteric plexuses with plates of endings (the "intraganglionic laminar endings") and infiltrating muscle sheets (the "intramuscular arrays" coursing with interstitial cells of Cajal) (fig 3). If this network of visceral afferents generates "axon responses" or local "axon reflexes" in addition to conventional afferent signals, as has been hypothesised, ${ }^{6}$ it would add additional dimensions to the organisation of the neural circuitry connecting brain, or central nervous system, and gut. 


\section{Discussion}

In summary, the traditional view of an autonomous ENS only tenuously tethered to the CNS was based on absence of morphological observation, on indirect inferences, and on calculations considering numbers of axons rather than the extent of telodendria. Direct structural observations with modern neural tracing techniques portray a different organisation. A rich network of vagal connections extends from the brain and nodose ganglia to interpenetrate the enteric plexuses. This circuitry suggests that the ENS and CNS are welded together in a neural network in which outflow from the CNS may have pervasive effects on ENS operations, and the ENS may have extensive inputs to the CNS by way of the vagal afferents.

1 Langley JN. Connexions of the enteric nerve cells. F Physiol (Lond) 1922;56:39.

2 Wood JD. Physiology of the enteric nervous system. In: Johnson LR, ed. Physiology of the gastrointestinal tract, 2nd edn. New York: Raven Press, 1987:670-109.
3 Kirchgessner AL, Gershon MD. Identification of vagal efferent fibers and putative target neurons in the enteric nervous system of the rat. F Comp Neurol 1989;285:38-53.

4 Holst M-C, Kelly JB, Powley TL. Vagal preganglionic projections to the enteric nervous system characterized with Phaseolus vulgaris-leucoagglutinin. I Comp Neurol 1997;381:81-100.

5 Berthoud H-R, Jedrzejewska A, Powley TL. Simultaneous identification of afferent inputs and efferent gastrointestinal projections of the dorsal vagal complex in the rat. f Comp Neurol 1990;301:65-79.

6 Berthoud H-R, Powley TL. Vagal afferent innervation of the rat fundic stomach: Morphological characterization of the gastric tension receptor. F Comp Neurol 1992;319:261-76.

7 Phillips RI, Baronowsky EA, Powley TL. Afferent innervation of gastrointestinal tract smooth muscle by the hepatic branch of the vagus. $\mathcal{F}$ Comp Neurol 1997;384:248-70.

8 Jarvinen MK, Powrozek TA, Wollmann WJ, et al. Specializations of vagal intraganglionic laminar endings (IGLEs) in the rat stomach and duodenum: A quantitative analysis. Proc Anпu Meet Soc Neurosci 1998;24:1123.

9 Powley TL, Holst M-C, Boyd DB, et al. 3-Dimensional reconstructions of autonomic projections to the gastrointestinal tract. Microsc Res Tech 1994;29:297-309.

10 Berthoud H-R, Carlson NR, Powley TL. Topography of efferent vagal innervation of the rat gastrointestinal tract. Am F Physiol 1991;260:R200-7.

11 Schemann M, Grundy D. Electrophysiological identification of vagally innervated enteric neurons in guinea pig stomach. Am f Physiol 1992;263:G709-18. 